\title{
Agricultura 4.0 aplicada à floricultura - PhenoGlad Mobile SC
}

Leosane Cristina Bosco'; Nereu Augusto Streck²; Lilian Osmari Uhlmann 3 ; Alexandra Goede de Souza4; Otavio Bagiotto Rossato ${ }^{5}$; Melina Inês Bonatto ${ }^{6}$; Lucas Ferreira da Silva ${ }^{7}$ e Rômulo Pulcinelli Benedetti ${ }^{7}$

Resumo - O planejamento da produção é essencial para garantir a qualidade de flores exigida pelo mercado consumidor. $O$ trabalho tem como objetivo descrever o funcionamento do aplicativo PhenoGlad Mobile SC, uma ferramenta digital para o planejamento do cultivo de gladíolo ou palma-de-santa-rita. O PhenoGlad Mobile SC propõe acessibilidade ao produtor rural, por apresentar interface amigável e funcionamento off-line depois de realizado o download do aplicativo. Com o aplicativo, podem ser obtidas simulações a partir da data de plantio ou de colheita das flores, possibilitando o planejamento de produção de gladíolo para todos os municípios de SC.

Termos para indexação: Gladiolus x grandiflorus Hort.; aplicativo móvel; planejamento da produção; Flores para Todos.

\section{Agriculture 4.0 applied to floriculture - The Phenoglad Mobile SC}

Abstract - Production planning is essential to guarantee the quality of flowers required by the consumer market. The work aims to describe the PhenoGlad Mobile SC App, a digital tool for planning the cultivation of gladiolus. PhenoGlad Mobile SC proposes accessibility to farmers, as it presents a friendly interface and offline operation after downloading. With this App, simulations can be obtained from the date of planting or from the date of harvest of flowers, enabling the production planning of gladiolus for all municipalities in SC.

Index terms: Gladiolus x grandiflorus Hort.; mobile App; production planning; Flowers for everyone

\section{Introdução}

A quarta revolução industrial representa a transformação de vários setores a partir de tecnologias digitais. Na agricultura 4.0 a disseminação de tecnologias móveis, serviços de sensoriamento remoto e computação está melhorando o acesso as informações pelos agricultores e criando novas oportunidades (USAID, 2018). A agricultura digital, portanto, tornou-se uma estratégia para adaptar o setor agrícola às mudanças tecnológicas estruturais tanto na produção quanto na gestão. No contexto dos objetivos do Desenvolvimento Sustentável da FAO, a agricultura digital tem o potencial de oferecer benefícios econômicos pelo aumento da produtividade e pela eficiência na produção, gerando benefícios ambientais através do uso otimizado de recursos. Além disso, são esperados benefícios sociais e culturais com o aumento da comunicação e, consequentemente, maior inclusão (TRENDOV et al., 2019).

O gladíolo é uma flor de corte cultivada a campo (Figura 1A) adaptada às condições edafoclimáticas de Santa Catarina, sendo considerada uma ótima opção para diversificação da produção e da renda agrícola (BONATTO, 2019; UHLMANN et al., 2019; SCHWAB et al., 2019). Levando em consideração o po- tencial de produção de flores de gladíolo em $\mathrm{SC}$ e as dificuldades de acesso às tecnologias no meio rural, principalmente na agricultura familiar, desenvolveu-se um aplicativo (app) com funcionamento totalmente off-line, de acesso livre, com o intuito de auxiliar no planejamento e nas tomadas de decisão para cultivo de gladíolo e, assim, reduzir riscos associados às adversidades climáticas.

O desenvolvimento desse app é o resultado da integração de unidades de ensino com grupos de pesquisa e extensão através das Equipes PhenoGlad que conduzem experimentos on farm no RS e SC. O aplicativo PhenoGlad Mobile RS (da SILVA et al., 2018) foi o precursor e

Recebido em 14/4/2020. Aceito para publicação em 22/6/2020.

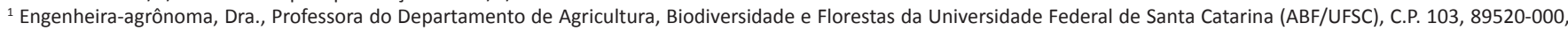
Curitibanos, SC, fone: (48) 3721-6454, e-mail: leosane.bosco@ufsc.br.

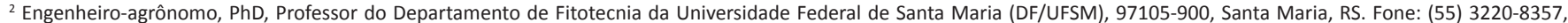

e-mail: nstreck2@yahoo.com.br.

${ }^{3}$ Engenheira-agrônoma, Dra., Professora DF/UFSM, e-mail: uhlmannlilian@gmail.com.

${ }^{4}$ Engenheira-agrônoma, Dra., Professora do Instituto Federal Catarinense (IFC), 89160-000, Rio do Sul, SC, fone: (47) 3531-3700, e-mail: alexandra.souza@ifc.edu.br.

${ }^{5}$ Engenheiro-agrônomo, Dr., Professor IFC, 89703-720, Concórdia, SC, fone: (49) 3441-4800, e-mail: otavio.rossato@ifc.edu.br.

${ }^{6}$ Engenheira-agrônoma, Msc., ABF/UFSC, e-mail: melinabonatto@gmail.com.

${ }^{7}$ Cientistas da Computação, DF/UFSM, e-mails: Iferreira@inf.ufsm.br; romuluspb@gmail.com. 


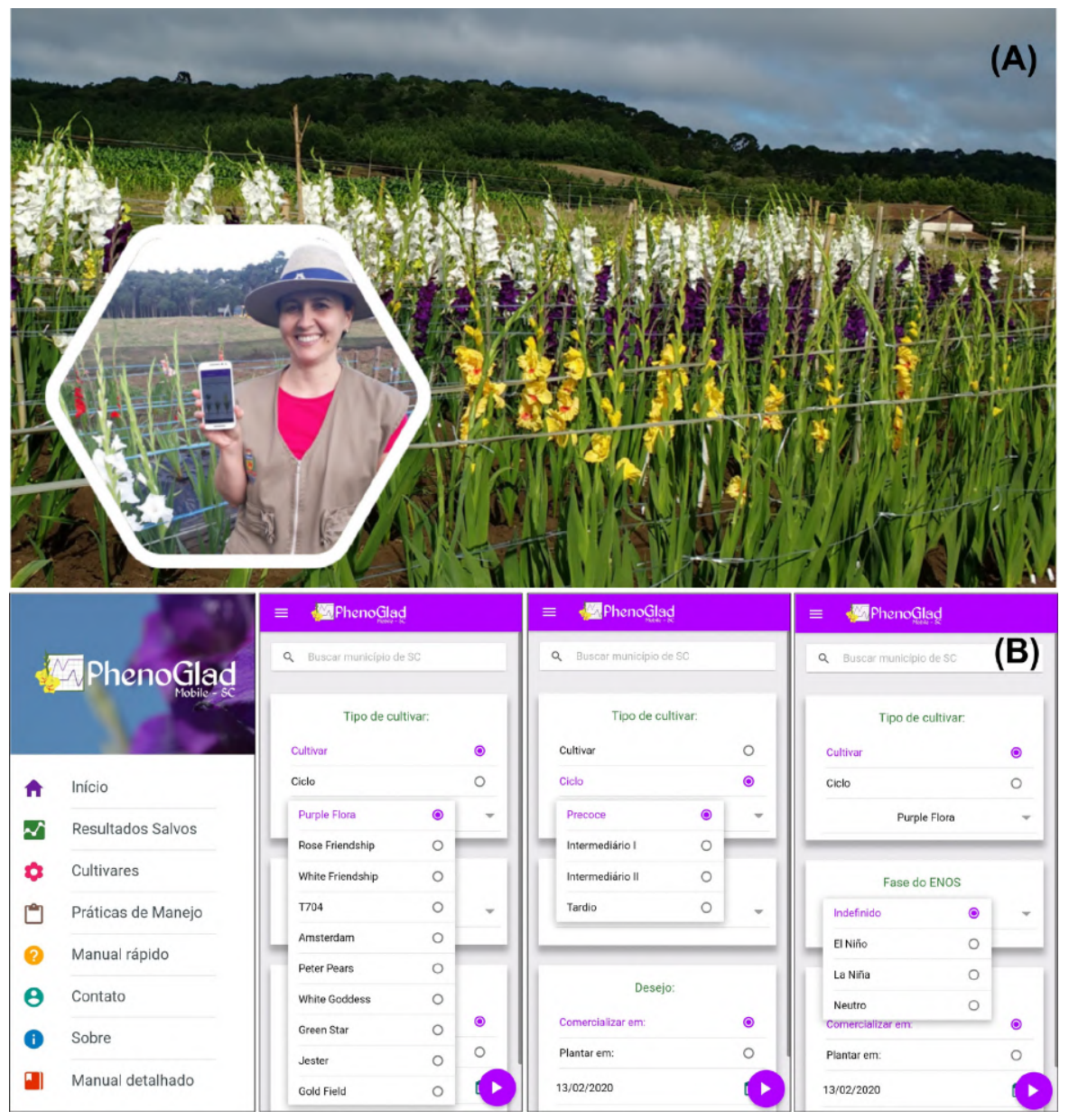

Figura 1. Área de gladíolos planejada com PhenoGlad Mobile SC (A) e interface do aplicativo (B). Autor: Leosane C. Bosco

Figure 1. Gladiolus area planned with PhenoGlad Mobile SC (A) and interface of the App (B). Author: Leosane C. Bosco

motivador para o desenvolvimento do PhenoGlad Mobile SC. Esses aplicativos vêm sendo usados desde 2018 por extensionistas, produtores e escolas do campo para o planejamento do cultivo de gladíolo visando à diversificação de culturas e de renda de agricultores (UHLMANN et al., 2019). É a principal ferramenta de manejo do gladíolo no projeto Flores para Todos, uma iniciativa da extensão rural que visa levar a floricultura como uma alternativa de renda para agricultores familiares e manter o jovem no campo. Desde seu início em 2018 até final de 2019, alcançou 55 municípios nos estados da Região Sul do Brasil e beneficiou 67 famílias e 10 escolas do campo, com produção de $48 \mathrm{mil}$ hastes florais de gladíolo.

O PhenoGlad Mobile SC realiza o cálculo do desenvolvimento do gladíolo do plantio até a colheita, a partir da escala fenológica e de dados históricos de temperaturas regionais. Os dados meteorológicos também foram agrupados conforme a Fase do El Niño Oscilação Sul (ENOS), pois esse evento modifica a temperatura do ar e pode alterar a duração do ciclo do gladíolo (BECKER et al., 2019).

O aplicativo foi desenvolvido em linguagem de programação HTML5, JavaScript, CSS, JSON e está disponível gratuitamente para download em dispositivos móveis com sistema operacional Android na Play Store.

Estudos realizados pela Equipe PhenoGlad SC nos municípios de Curitibanos, Concórdia e Rio do Sul vêm demonstrando a capacidade de predição da data de colheita pelo software (BONATTO, 2019).

\section{Funcionalidades do}

\section{aplicativo}

$\mathrm{Na}$ interface do aplicativo encontram-se informações relacionadas a cultivares, práticas de manejo, resultados salvos, além de manuais e contato da equipe responsável para auxiliar o usuário (Figura 1B). Na tela principal há a opção de escolha do município em que será realizado o plantio, o cultivar desejado ou, caso o cultivar não estiver elencado na lista, há a opção de escolha da duração do ciclo das plantas. Para a realização do cálculo é necessário indicar a fase do El Niño Oscilação Sul (ENOS) referente ao ano em que deseja realizar o cultivo (Figura 1B). Quando o usuário selecionar a fase denominada de "Indefinido", por não ter a informação sobre a condição do ENOS, o aplicativo irá considerar a média histórica de todos os anos de dados. Para as demais fases do ENOS (El Niño, La Niña e Neutro), a média histórica foi realizada considerando os anos em que a respectiva fase se configurou.

O passo seguinte é a seleção da data em que o plantio será realizado ou da data prevista para a comercialização. Se o usuário quiser realizar o plantio em uma data específica e desejar saber a data em que as hastes florais ficarão prontas para serem comercializadas, o aplicativo gerará a data provável da coIheita, ou seja, do estágio R3.4, quando metade dos floretes estão abertos. No exemplo da Figura 2A, o plantio do cultivar Amsterdam em Florianópolis será realizado no dia $17 / 03$, sendo a colheita estimada para 29/05. Se o usuário quiser comercializar as hastes em uma data específica e desejar saber a data em que o plantio dos cormos deverá ser realizado, o aplicativo mostrará qual data de plantio que resultará no estágio R3.4. Caso o produtor receba uma encomenda para uma festa ou queira ter flores para comercialização em datas de pico de vendas, como Dia de Finados, por exemplo, ele pode fazer a programação da data de plantio baseando-se na data de venda das hastes florais. No exemplo 


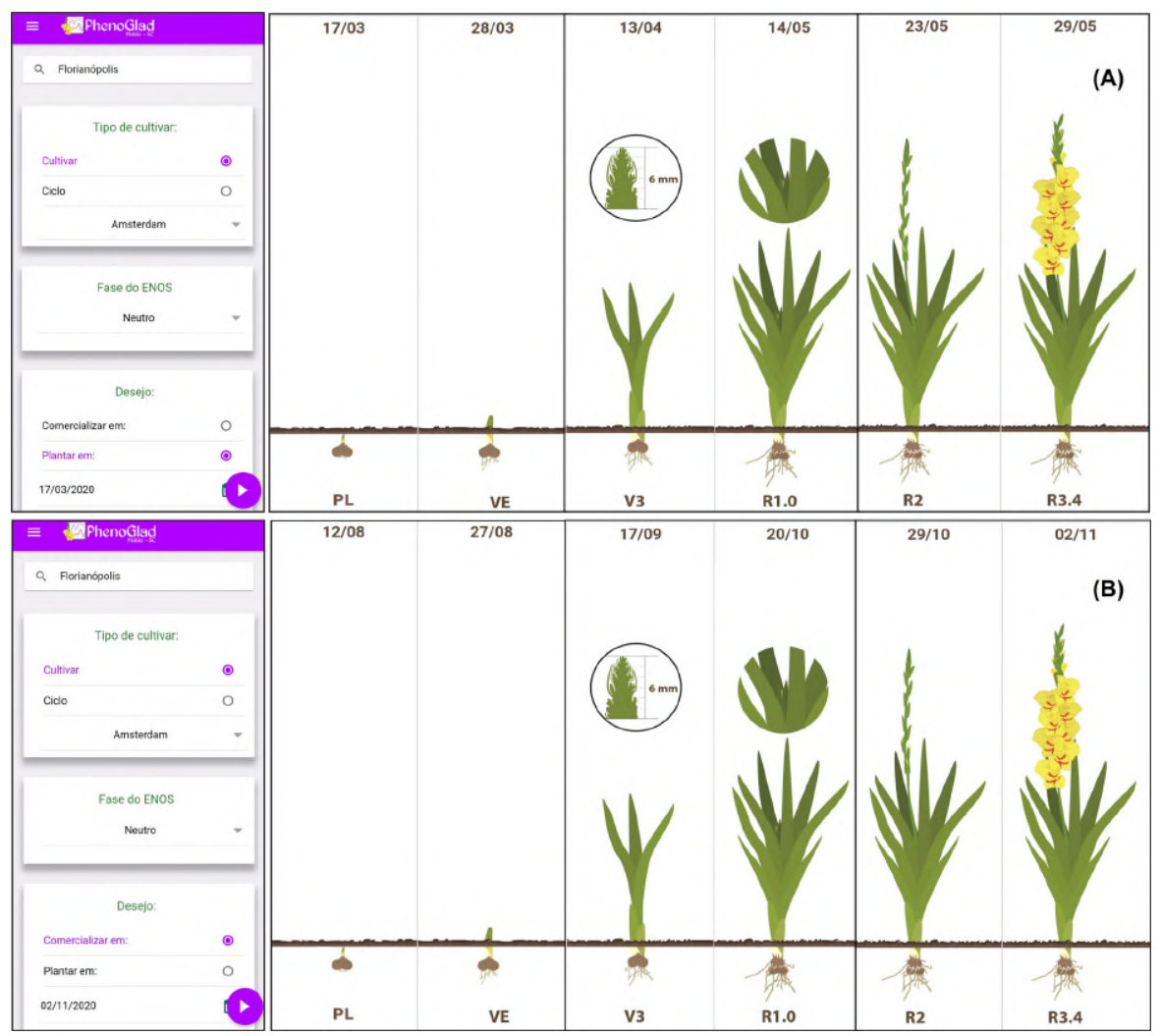

Figura 2. Seleção da data em que o plantio será realizado $(A)$ ou da data prevista para a comercialização (B) com demonstração dos resultados. Exemplo considerado para o município de Florianópolis, SC com o cultivar Amsterdam. Autor: Leosane C. Bosco Figure 2. Selection of data on which planting will be carried out (A) or data expected for sale (B) with a statement of results. Example considered for Florianópolis, SC, with the cultivar Amsterdam. Author: Leosane C. Bosco

da Figura 2B, o plantio será em 12/08 para que o produtor tenha flores para venda no dia 02/11.

Após a seleção de todos os dados necessários para a simulação é preciso pressionar o botão de cor roxa para obtenção dos resultados. As simulações são salvas na aba "Resultados Salvos", localizada na interface do aplicativo (Figura 1).

\section{Alertas gerados pelo aplicativo}

Com relação aos possíveis estresses da cultura, destaca-se o déficit hídrico e as temperaturas extremas. A fenologia da cultura do gladíolo calculada no PhenoGlad Mobile não leva em consideração a falta ou o excesso de água no solo, ou seja, o aplicativo informa o usuário, na aba práticas de manejo, que deve ser ras. Nesse caso, uma recomendação prática é emitida, informando a necessidade de utilização de tela de sombreamento sobre as plantas a partir da emissão da espiga, para evitar esses danos.

$\mathrm{O}$ dano de morte da espiga por geada em gladíolo é considerado no PhenoGlad Mobile quando a temperatura mínima (Tmin) é menor a $-2^{\circ} \mathrm{C}$ por um dia ou $2^{\circ} \mathrm{C} \leq \operatorname{Tmin} \leq 3^{\circ} \mathrm{C}$ durante 3 dias consecutivos durante a fase reprodutiva da cultura. Nesses casos, não há morte das folhas, mas elas são levemente danificadas. Caso o usuário selecione a data de plantio ou a data de comercialização desejadas, e durante o período de cultivo resultar em uma data de plantio que historicamente causa morte da espiga por baixas temperaturas, o aplicativo emite um alerta com a seguinte informação: Esta data de plantio não é recomendada para este local, pois há risco de morte da espiga pela ocorrência de geada.

O PhenoGlad Mobile SC simula possíveis danos ocasionados pelas condições climáticas da região, indicando datas de plantio não recomendadas e orientando o agricultor com indicações de práticas de manejo para mitigar os danos. Na Figura 3 pode-se observar um estudo de caso para o município de Curitibanos considerando-se um plantio em 17/03 e para São Miguel do Oeste com plantio em 16/12. Em regiões com invernos característicos de clima temperado, como Curitibanos, ou com verões quentes, como em São Miguel do Oeste, não se recomenda o cultivo em algumas épocas devido a danos nas plantas, principalmente na fase reprodutiva (UHLMANN et al., 2017).

\section{Considerações finais}

Os danos por altas temperaturas ocorrem quando a temperatura máxima diária do ar é maior ou igual a $34^{\circ} \mathrm{C}$ por no mínimo três dias consecutivos. Se a fase reprodutiva da cultura ocorrer nos meses de novembro, dezembro, janeiro, fevereiro ou março, o aplicativo avisa o usuário sobre o risco de queimaduras nas hastes florais por altas temperatu- 


\section{$\equiv \quad$ Práticas de Manejo}

\section{Orrigação}

A irrigação da lavoura deve ser realizada, preferencialmente, com sistema de gotejamento para evitar o molhamento da parte aérea. Irrigar a lavoura sempre que necessário para evitar o ressecamento do solo e o déficit hídrico das plantas. Tomar cuidado para não deixar o solo excessivamente úmido, pois pode causar o apodrecimento dos bulbos.
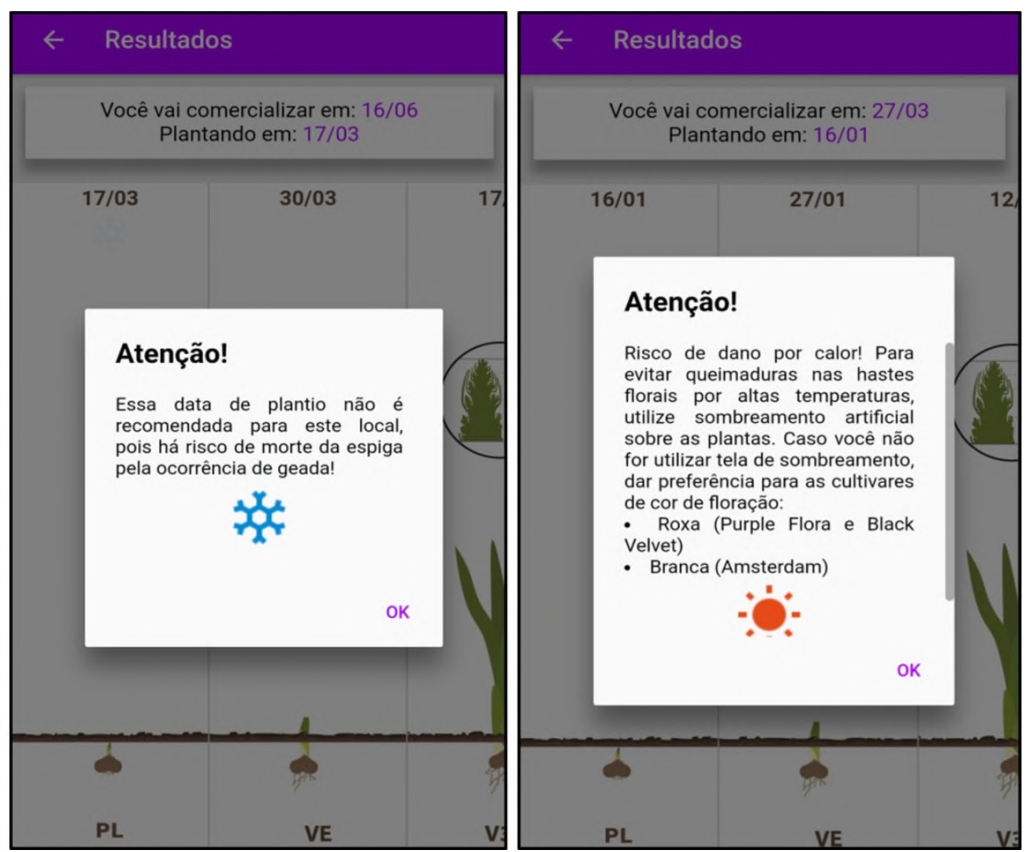

Figura 3. Descrição da prática de manejo irrigação e simulações que resultaram em alertas de danos ocasionados pelo frio em Curitibanos, SC, com plantio em 17/03, e por calor em São Miguel do Oeste, SC, com plantio em 16/01. Autor: Leosane C. Bosco

Figure 3. Description of management practice Irrigation and simulations that resulted in warnings of damage caused by the cold in Curitibanos, SC with planting on 17 March and by heat in São Miguel do Oeste, SC with planting on 16 January. Author: Leosane C. Bosco

nhecidos já utilizaram essa ferramenta para planejamento da produção. Além disso, o app está em constante atualização e sendo utilizado em atividades de ensino, pesquisa e extensão em SC. 0 projeto de extensão Flores para Todos utiliza o aplicativo para dar orientações e suporte aos produtores de gladíolo que estão sendo atendidos pela Equipe PhenoGlad SC.

Publicações das aplicações práticas do PhenoGlad Mobile estão sendo realizadas constantemente nas redes sociais oficiais da Equipe PhenoGlad:

www.facebook.com/phenoglad, www.instagram.com/phenoglad,

www.twitter.com/phenoglad e no canal do YouTube PhenoGlad.

\section{Referências}

BECKER, C.C.; STRECK, N.A; UHLMANN, L.O.; SILVEIRA, W.B. Scheduling optimum planting window for gladiola based on El Niño Southern Oscillation. Scientia Agricola, v.77, n.5, e20180336, 2019. DOI: http://dx.doi. org/10.1590/1678-992x-2018-0336
BONATTO, M.I. Análise de riscos climáticos para o cultivo do gladíolo em Santa Catarina, Sul do Brasil. 2019. 103f. Dissertação (Mestrado em Ciências) - Universidade Federal de Santa Catarina, Curitibanos, SC, 2019. Disponível em: https://repositorio. ufsc.br/handle/123456789/204515. Acesso em: 26 fevereiro 2020.

da SILVA, L.F.; UHLMANN, L.O., BECKER, C.C.; STRECK, N.A; CHARÃO, A.S.; TOMIOZZO, R. PhenoGlad Mobile: um aplicativo para simulação da fenologia em gladíolo. Revista Brasileira de Iniciação Científica, v.5, n.6, p.117-132, 2018. Disponível em: https:// periodicos.itp.ifsp.edu.br/index.php/IC/article/view/1345. Acesso em: 20 fev. 2020.

SCHWAB, N.T.; UHLMANN, L.O.; BECKER, C.C.; TOMIOZZO, R.; STRECK, N.A.; BOSCO, L.C.; BONATTO, M.I.; STANCK, L.T. Gladíolo - Fenologia e manejo para produção de hastes e bulbos. 1.ed. Santa Maria: [S.N.], 2019. 136p.

TRENDOV, N.M.; VARAS, S.; ZENG, M. Digital technologies in agriculture and rural areas. Briefing paper. Rome: Food and Agriculture Organization of the United Nations, 2019. 26p. ISBN 978-92-5-131546-0. Disponível em: http://www.fao.org/3/ca4887en/ca4887en.pdf. Acesso em: 26 fev. 2020.

UHLMANN, L.O.; STRECK, N.A.; BECKER, C.C.; SCHWAB, N.T.; BENEDETTI, R.P.; CHARÃO, A.S.; RIBEIRO, B.S.M.R.; SILVEIRA, W.B.; BACKES, F.A.A.L.; ALBERTO, C.M.; MUTTONI, M.; PAULA, G.M. de; TOMIOZZO, R.; BOSCO, L.C.; BECKER, D. PhenoGlad: a model for simulating development in Gladiolus. European Journal of Agronomy, v.82, p.3349, 2017. DOI: https://doi.org/10.1016/j. eja.2016.10.001

UHLMANN, L.O.; BECKER, C.C.; TOMIOZZO, R.; STRECK, N.A.; SCHONS, A.; BALEST, D.S.; BRAGA, M.S.; SCHWAB, N.T.; LANGNER, J.A. Gladiolus as an alternative for diversification and profit in small rural property. Ornamental Horticulture, v.25, p.200-208, 2019. DOI: http://dx.doi.org/10.14295/oh.v25i2.1541

USAID - United State Agency International Development. 2018. Digital farmer profile: Reimagining Smallholder Agriculture. Washington D.C.: USAID. 84p. Disponível em: https://grameenfoundation.org/partners/ resources/digital-farmer-profiles-reimagining-smallholder-agriculture. Acesso em: 20 mar. 2020. 